\title{
Replacing carbohydrate during a glucose challenge with the egg white portion or whole eggs protects against postprandial impairments in vascular endothelial function in prediabetic men by limiting increases in glycaemia and lipid peroxidation
}

Joshua D. McDonald ${ }^{1}$, Chureeporn Chitchumroonchokchai ${ }^{1}$, Jinhui $\mathrm{Li}^{1}$, Eunice Mah ${ }^{1,2}$, Allison N. Labyk ${ }^{1}$, Elizabeth J. Reverri ${ }^{1}$, Kevin D. Ballard ${ }^{3}$, Jeff S. Volek ${ }^{4}$ and Richard S. Bruno ${ }^{1 *}$

${ }^{1}$ Human Nutrition Program, Department of Human Sciences, The Obio State University, Columbus, OH 43210, USA

${ }^{2}$ Biofortis, Inc., Addison, IL 60101, USA

${ }^{3}$ Department of Kinesiology and Health, Miami University, Oxford, OH 45056, USA

${ }^{4}$ Kinesiology Program, Department of Human Sciences, The Obio State University, Columbus, OH 43210, USA

(Submitted 10 June 2017 - Final revision received 30 October 2017 - Accepted 22 November 2017 - First published online 16 January 2018)

\section{Abstract}

Eggs attenuate postprandial hyperglycaemia (PPH), which transiently impairs vascular endothelial function (VEF). We hypothesised that co-ingestion of a glucose challenge with egg-based meals would protect against glucose-induced impairments in VEF by attenuating PPH and oxidative stress. A randomised, cross-over study was conducted in prediabetic men $(n$ 20) who ingested isoenegertic meals $(1674 \mathrm{~kJ}$ (400 kcal)) containing $100 \mathrm{~g}$ glucose (GLU), or $75 \mathrm{~g}$ glucose with 1.5 whole eggs (EGG), seven egg whites (WHITE) or two egg yolks (YOLK). At $30 \mathrm{~min}$ intervals for $3 \mathrm{~h}$, brachial artery flow-mediated dilation (FMD), plasma glucose, insulin, cholecystokinin (CCK), lipids (total, LDL- and HDL-cholesterol; TAG), $\mathrm{F}_{2}$-isoprostanes normalised to arachidonic acid ( $\mathrm{F}_{2}$-IsoPs/AA), and methylglyoxal were assessed. In GLU, FMD decreased at 30-60 min and returned to baseline levels by $90 \mathrm{~min}$. GLU-mediated decreases in FMD were attenuated at 30-60 min in EGG and WHITE. Compared with GLU, FMD ${ }_{\mathrm{AUC}}$ was higher in EGG and WHITE only. Relative to baseline, glucose increased at 30-120 min in GLU and YOLK but only at 30-90 min in EGG and WHITE. Glucose $\mathrm{AUC}_{\mathrm{AUC}}$ and insulin AUC $_{\text {were }}$ also lower in EGG and WHITE only. However, $\mathrm{CCK}_{\mathrm{AUC}}$ was higher in EGG and WHITE compared with GLU. Compared with GLU, $\mathrm{F}_{2}$-IsoPs/AA $\mathrm{AUC}_{\mathrm{AUC}}$ was lower in EGG and WHITE but unaffected by YOLK. Postprandial lipids and methylglyoxal did not differ between treatments. Thus, replacing a portion of a glucose challenge with whole eggs or egg whites, but not yolks, limits postprandial impairments in VEF by attenuating increases in glycaemia and lipid peroxidation.

Key words: Eggs: Postprandial hyperglycaemia: Gastric emptying: Oxidative stress: Vascular function

CVD has been the leading cause of mortality in the USA for nearly a century ${ }^{(1)}$. Although numerous risk factors are implicated, epidemiological evidence supports that the magnitude of postprandial hyperglycaemia (PPH) is an independent predictor of CVD-related mortality ${ }^{(2)}$. The mechanisms by which PPH induce CVD are under investigation $^{(3,4)}$. PPH transiently impairs vascular endothelial function $(\mathrm{VEF})$ in an oxidative stress-dependent manner that limits the bioavailability of nitric oxide $\left(\mathrm{NO}^{\circ}\right)^{(5)}$, an endothelial-derived vasodilator that regulates vascular homoeostasis ${ }^{(6)}$. Dietary modification is a leading strategy to limit PPH-mediated impairments in VEF either by attenuating PPH and/or limiting downstream oxidative stress responses that induce vascular injury.

$\mathrm{PPH}$ following an oral glucose challenge induces oxidative stress as evidenced by increases in the lipid peroxidation biomarkers malondialdehyde ${ }^{(5)}$ and $\mathrm{F}_{2}$-isoprostanes $\left(\mathrm{F}_{2}\right.$-IsoPs ${ }^{(7)}$.
$\mathrm{F}_{2}$-IsoPs are non-enzymatic oxidation products of arachidonic acid $(\mathrm{AA})^{(7,8)}$ and specific stereoisomers, such as 8-iso-PG $\mathrm{F}_{2 \alpha}$ (8-iso-PGF ${ }_{2 \alpha}$ ), are well associated with $\mathrm{CVD}^{(9)}$. $\mathrm{F}_{2}$-IsoPs contribute to CVD by increasing platelet aggregation and immune cell adhesion and inducing vasoconstriction ${ }^{(9)}$. Separate from this, PPH increases the formation of methylglyoxal (MGO), a precursor to advanced glycation end products that provokes inflammation and oxidative stress ${ }^{(10)}$, which would be expected to contribute to impairments in VEF.

Brachial artery flow-mediated dilation (FMD) is a noninvasive ultrasound measurement of endothelial nitric oxide synthase (eNOS)-mediated, $\mathrm{NO}^{\circ}$-dependent vasodilation that assesses VEF and CVD risk ${ }^{(11,12)}$. In healthy men following an oral glucose challenge, PPH was positively correlated with lipid peroxidation and both $\mathrm{PPH}$ and lipid peroxidation were

Abbreviations: AA, arachidonic acid; CCK, cholecystokinin; EGG, whole eggs; $\mathrm{F}_{2}$-IsoPs, $\mathrm{F}_{2}$-isoprostanes; FMD, flow-mediated dilation; GLU, glucose; MGO, methylglyoxal; NO', nitric oxide; PPH, postprandial hyperglycaemia; VEF, vascular endothelial function; WHITE, egg whites; YOLK, egg yolks.

* Corresponding author: R. S. Bruno, fax +1614292 4339, email bruno.27@osu.edu 
inversely correlated with FMD responses ${ }^{(5,13)}$. These data indicate that postprandial increases in blood glucose are associated with increases in oxidative stress and decreases in vascular function. Indeed, PPH-mediated oxidative stress is implicated in impairing VEF by reducing $\mathrm{NO}^{\bullet}$ bioavailability ${ }^{(5)}$.

Findings from observational studies are equivocal regarding egg consumption in relation to CVD risk $^{(14-17)}$, with some suggesting neutral or even adverse effects on cardiovascular health. In contrast, controlled studies indicate favourable metabolic outcomes of egg consumption on PPH-mediated CVD risk ${ }^{(18-22)}$. Chronic consumption of eggs improves insulin sensitivity $^{(18)}$, which would be expected to promote tissue glucose uptake. In healthy men, consumption of whole eggs and egg yolks, when added to a controlled meal ( $6 \%$ protein, $49 \%$ fat, $45 \%$ carbohydrate) increased circulating cholecystokinin $(\mathrm{CCK})^{(22)}$, a gastrointestinal hormone that functions to delay gastric empting. This potentially slowed glucose absorption to limit $\mathrm{PPH}^{(22)}$. Chronic ingestion of eggs also attenuates lipid peroxidation ${ }^{(18,23)}$ and neither their acute nor chronic ingestion impairs $\mathrm{VEF}^{(19-21)}$. However, no postprandial studies have examined the different fractions of eggs on gut-level CCK responses to limit $\mathrm{PPH}$-mediated oxidative stress leading to impairments in VEF. We hypothesised that co-ingestion of a glucose challenge with egg-based meals would protect against glucose-induced impairments in VEF consistent with a mechanism of delayed gastric emptying that limits $\mathrm{PPH}$ and downstream lipid peroxidation. To test this, prediabetic men completed a four-arm, randomised, cross-over trial in which they ingested isoenergetic meals of glucose alone or in combination with egg-based meals. We then assessed VEF and biomarkers of oxidative stress and cardiometabolic health during the 3-h postprandial period.

\section{Methods \\ Participants}

Men with prediabetes ( $n$ 20) were enrolled on the basis of age (25-50 years), BMI between 25 and $35 \mathrm{~kg} / \mathrm{m}^{2}$, total cholesterol $<6.2 \mathrm{mmol} / 1$, and resting systolic and diastolic blood pressure of $\leq 140 \mathrm{mmHg}$ and $\leq 90 \mathrm{mmHg}$, respectively. Middle-aged men were specifically enrolled to circumvent confounding factors of age and sex on FMD responses ${ }^{(12)}$. Participants were required to have fasting blood glucose between 5.6 and $6.9 \mathrm{mmol} / \mathrm{l}$ according to established criteria for prediabetes ${ }^{(24)}$. They were also required to be non-vegetarian, non-smokers, non-users of dietary supplements or vasoactive medications ( $>1$ month), participate in $<7 \mathrm{~h} /$ week of aerobic exercise, be weight stable ( $\pm 2 \mathrm{~kg}$ for 3 months), consume $<3$ alcoholic drinks/d, and selfreported to have no known history of CVD.

\section{Chemicals and reagents}

HPLC-grade solvents and the following chemicals were purchased from Fisher Scientific: acetic acid, formic acid, hexane, hydrochloric acid $(\mathrm{HCl})$, methanol, sodium hydroxide $(\mathrm{NaOH})$, acetonitrile, perchloric acid (PCA). 5-methylquinoxaline (5-MQ), $o$-phenylenediamine (OPD) and MGO were purchased from
Sigma-Aldrich. Unlabelled $\left(\mathrm{d}_{0^{-}}\right)$and octa- ${ }^{2} \mathrm{H}$-labelled $\left(\mathrm{d}_{8^{-}}\right)$AA were purchased from Cayman Chemical.

\section{Study design}

The present study was conducted according to the guidelines laid down in the Declaration of Helsinki and all procedures involving human subjects were approved by the Institutional Review Board at The Ohio State University (2014H0307). Written informed consent was obtained from all subjects before enrolling (Fig. 1). This study was registered at ClinicalTrials.gov (NCT02364570). Participants completed a four-arm, randomised, cross-over trial in which they visited the study centre after an overnight fast (10-12 h) on four occasions separated by a washout period of at least $7 \mathrm{~d}$. During each visit, participants ingested isoenergetic meals (about $1674 \mathrm{~kJ}$ (400 kcal); Table 1) of either glucose alone (GLU; $100 \mathrm{~g}$ in $296 \mathrm{ml}$ water, Trutol ${ }^{\circledR}$; Thermo Scientific), or glucose ( $75 \mathrm{~g}$ in $296 \mathrm{ml}$ water) in combination with 1.5 whole eggs (EGG), seven egg whites (WHITE) or two egg yolks (YOLK). Eggs were purchased (Giant Eagle) and egg-based meals were scrambled without any additional ingredients and frozen based on mass into single-serve portions at the beginning of the study. Portioned meals were thawed overnight in a ceramic container and microwaved immediately before ingestion. All meals were consumed within $10 \mathrm{~min}$. Each test meal provided participants with a typical energy content of breakfast for American men ${ }^{(25)}$. The amount of glucose provided in the eggbased meals $(75 \mathrm{~g}$ ) is equivalent to that of an oral glucose tolerance test, and has been demonstrated to induce $\mathrm{PPH}$, oxidative stress, and impairments in VEF by our group ${ }^{(5)}$. Blood samples were collected before test meal ingestion at baseline $(t=0 \mathrm{~min})$ of each trial and at $30 \mathrm{~min}$ intervals during the 3 -h postprandial period. Brachial artery FMD, blood pressure, and heart rate were also measured at these time points. Participants' blood pressure and heart rate were measured using an automated blood pressure monitor (Omron BP760; Omron Healthcare, Inc.).

\section{Sample handling}

Venous blood samples were collected into evacuated tubes containing EDTA or sodium heparin from a flexible catheter inserted into the antecubital vein of the left arm. Plasma was obtained following centrifugation $\left(4^{\circ} \mathrm{C}, 15 \mathrm{~min}, 1500 \mathrm{~g}\right)$,

Table 1. Energy, mass and macronutrient content of study test meals (Numbers and percentages of energy for each macronutrient within each meal)

\begin{tabular}{|c|c|c|c|c|c|c|c|c|}
\hline & \multicolumn{2}{|c|}{ GLU } & \multicolumn{2}{|c|}{ EGG } & \multicolumn{2}{|c|}{ WHITE } & \multicolumn{2}{|c|}{ YOLK } \\
\hline & $n$ & $\%$ & $n$ & $\%$ & $n$ & $\%$ & $n$ & $\%$ \\
\hline Energy (kJ) & \multicolumn{2}{|c|}{1674} & \multicolumn{2}{|c|}{1686} & \multicolumn{2}{|c|}{1678} & \multicolumn{2}{|c|}{1686} \\
\hline Energy (kcal) & \multicolumn{2}{|c|}{400} & \multicolumn{2}{|c|}{403} & \multicolumn{2}{|c|}{401} & \multicolumn{2}{|c|}{403} \\
\hline Mass (g) & \multicolumn{2}{|c|}{296} & \multicolumn{2}{|c|}{371} & \multicolumn{2}{|c|}{527} & \multicolumn{2}{|c|}{330} \\
\hline Carbohydrate (g) & 100 & 100 & 75 & 75 & 75 & 75 & 75 & 75 \\
\hline Protein $(\mathrm{g})$ & 0 & 0 & 9.5 & 10 & 25 & 25 & 5 & 5 \\
\hline Fat $(g)$ & 0 & 0 & 7 & 15 & 0 & 0 & 9 & 20 \\
\hline
\end{tabular}

GLU, glucose; EGG, whole eggs; WHITE, egg whites; YOLK, egg yolks. 
aliquoted into cryovials, snap-frozen in liquid $\mathrm{N}_{2}$, and stored at $-80^{\circ} \mathrm{C}$ until analysis.

\section{Dietary modifications and analysis}

Participants were instructed by a registered dietitian to consume identical euenergetic diets, free of major sources of eggs, for each 3-d period before each trial. They were also instructed to abstain from exercise, caffeine and alcohol for $48 \mathrm{~h}$ before each trial to prevent their confounding effects on FMD responses ${ }^{(12)}$. Energy and nutrient intakes were assessed by 3-d food records before each trial and were analysed using Nutrition Data System for Research (University of Minnesota, NDSR 2015).

\section{Ultrasound measures of vascular health}

Carotid intima-media thickness (cIMT), an index of atherosclerosis risk, was assessed at baseline of each trial as described $^{(26)}$. In brief, a 13-MHz ultrasound transducer connected to a high-resolution T300 ultrasound system (Terason) was used to image the far wall of both the left and right common carotid arteries. Images were recorded for $10 \mathrm{~s}$ and analysed using edge-detection software (Carotid Analyzer for Research, Medical Imaging Applications). cIMT did not differ $(P>0.05)$ among trials and mean values are reported.

FMD was assessed using high-frequency ultrasound as we described $^{(5,13)}$. In brief, the brachial artery of the right arm was visualised by placing the transducer above the antecubital crease using the aforementioned ultrasound system. Preocclusion brachial artery diameter was recorded for $1 \mathrm{~min}$, after which an automated blood pressure cuff (Hokanson E20; Hokanson, Inc.) placed immediately distal to the olecranon process of the right arm was rapidly inflated to perform lower arm occlusion (200 mmHg, $5 \mathrm{~min}$ ). Post-occlusion vessel diameter recordings were initiated $1 \mathrm{~min}$ before cuff deflation and for $3 \mathrm{~min}$ thereafter. Pre- and post-occlusion images were analysed using edge-detection software (Medical Imaging Applications). Brachial artery FMD (\%) was calculated as: (peak post-occlusion diameter $(\mathrm{mm})$ - pre-occlusion diameter $(\mathrm{mm}))$ / pre-occlusion diameter $(\mathrm{mm}) \times 100$. All FMD measurements were performed by the same technician and images were analysed in a blinded manner

\section{Clinical chemistries}

Plasma glucose, total cholesterol, HDL-cholesterol, and TAG were measured according to the manufacturer's instructions (Pointe Scientific) using a UV2600 spectrophotometer (Shimadzu). LDL-cholesterol was calculated according to the Friedwald equation ${ }^{(27)}$. Plasma insulin (ALPCO) and CCK (LifeSpan Biosciences, Inc.) were measured by ELISA according to the manufacturers' instructions using a Synergy H1 microplate reader (Biotek Instruments). The homoeostatic model assessment of insulin resistance (HOMA-IR) was calculated from plasma collected at baseline $(t=0 \mathrm{~min})$ of each invention day as follows: plasma insulin $(\mu \mathrm{IU} / \mathrm{ml}) \times$ plasma glucose $(\mathrm{mmol} / \mathrm{l}) / 22 \cdot 5^{(28)}$. HOMA-IR did not differ $(P>0 \cdot 05)$ among trials and mean values are reported. Plasma $\mathrm{F}_{2}$-IsoPs were assessed by ELISA by measuring levels of 8-iso- $\mathrm{PGF}_{2 \alpha}$ according to the manufacturer's instructions (Enzo Life Sciences, Inc.).

\section{Plasma arachidonic acid}

To better define postprandial alterations in $\mathrm{F}_{2}$-IsoPs, plasma AA was measured as described ${ }^{(29)}$, with modifications to use an LCMS-2020 instrument (Shimadzu). In brief, $100 \mu$ of plasma was mixed with $900 \mu \mathrm{l}$ of water and $1 \mathrm{ml}$ of $1 \mathrm{~m} \mathrm{NaOH}$ in methanol before saponification $\left(60^{\circ} \mathrm{C}, 30 \mathrm{~min}\right)$. The saponified sample was acidified to $\mathrm{pH} 2 \cdot 85-3 \cdot 0$ using $600 \mu \mathrm{l}$ of $3 \mathrm{~m}-\mathrm{HCl}$ and $15 \mu \mathrm{l}_{8}$-AA $(50 \mu \mathrm{m}$ final concentration) was added as internal standard. The mixture was extracted with hexane and centrifuged $\left(2500 \mathrm{~g}, 10 \mathrm{~min}, 25^{\circ} \mathrm{C}\right)$ and supernatant was dried under $\mathrm{N}_{2}$ gas before resolubilisation with methanol containing $0 \cdot 1 \%$ formic acid. Samples were then injected onto the liquid chromatography-MS system equipped with an autosampler maintained at $4^{\circ} \mathrm{C}$ (SIL-20AC), a degassing unit (DGU-20A5), a column oven set to $30^{\circ} \mathrm{C}$ (CTO-20A) and two LC-30AD pumps. Instrument control was performed using Shimadzu LabSolution (version 5.7). Samples were separated on a Kinetex $\mathrm{C}_{18}$ column $(100 \times 2.1 \mathrm{~mm}, 2.6 \mu \mathrm{m}$; Phenomenex $)$ using an isocratic flow rate of $0.25 \mathrm{ml} / \mathrm{min}$ and mobile phase consisting of methanol containing $0.05 \%$ acetic acid. Nebulising and drying gases were supplied at 1.5 and 15 litres/m, and block and desolvation line temperatures were 450 and $300^{\circ} \mathrm{C}$, respectively. Detection was performed using single-ion monitoring following electrospray ionisation performed in negative mode. Endogenous AA ( $\mathrm{d}_{0}$-AA) and $\mathrm{d}_{8}$-AA (internal standard) were quantified at a mass:charge ratio $(\mathrm{m} / \mathrm{z})$ of 303 and 310 , respectively.

\section{Plasma methylglyoxal}

Plasma MGO was measured using HPLC-UV as described ${ }^{(30)}$, with minor modifications. In brief, MGO standard or $500 \mu \mathrm{l}$ of EDTA plasma was mixed with $100 \mu \mathrm{l} 2 \cdot 8 \mathrm{M}$ PCA and $30 \mu \mathrm{l}$ $212.68 \mathrm{~mm}$ OPD, respectively; $4 \mu \mathrm{l}$ 5-MQ (11.5 nm final concentration) was added as an internal standard. Following incubation $\left(25^{\circ} \mathrm{C}\right.$ in the dark for $\left.24 \mathrm{~h}\right)$ in a shaking water bath to derivatise $\mathrm{MGO}$ to 2-methylquinoxaline (2-MQ), samples were centrifuged $\left(10 \mathrm{~min}, 15000 \mathrm{~g}, 4^{\circ} \mathrm{C}\right)$ and the supernatant filtered $(0.22 \mu \mathrm{m})$ before injecting onto a Waters Alliance 2965 HPLC system. 2-MQ and 5-MQ were separated at $1.0 \mathrm{ml} / \mathrm{min}$ on a Nov-Pak $\mathrm{C}_{18}$ column $(150 \times 3.9 \mathrm{~mm}, 4 \mu \mathrm{m}$; Waters $)$ using a binary gradient of mobile phase A ( $20 \%$ acetonitrile in water) and mobile phase B (100\% methanol) as follows: 0-12 min, $0 \% \mathrm{~B}, 12-14 \mathrm{~min}, 80 \% \mathrm{~B}, 14-16 \mathrm{~min}, 100 \% \mathrm{~B}$, and $16-20 \mathrm{~min}$, $0 \%$ B. 2-MQ and 5-MQ were detected at $315 \mathrm{~nm}$ and quantified using peak area relative to internal standard based on a standard curve prepared in parallel.

\section{Statistical methods}

Sample size was determined using data from previous work examining postprandial changes in FMD responses following an oral glucose challenge ${ }^{(5)}$. Our power calculation indicated a minimum of nine subjects were needed to reject the null hypothesis with $90 \%$ power $(P<0.05)$. Data are expressed as means with their standard errors and were analysed using 
GraphPad Prism (version 7). Data reported are change from baseline $(\Delta)$ to better visualise between-treatment effects during the postprandial period. Time, treatment and time $\times$ treatment interaction effects for postprandial responses for FMD and plasma biomarkers were evaluated using two-way repeatedmeasures (RM) ANOVA with Bonferroni's correction to evaluate pairwise differences. AUC during the $180 \mathrm{~min}$ postprandial period $\left(\mathrm{AUC}_{0-180 \mathrm{~min}}\right)$ was calculated using the trapezoidal rule. Between-trial effects on dietary intakes, postprandial $\mathrm{AUC}_{0-180 \mathrm{~min}}$, and baseline values were evaluated using oneway RM ANOVA with Bonferroni's post test. Correlation coefficients $(r)$ were calculated using multiple linear regression while controlling for within-subject $\mathrm{RM}^{(31)} . P<0.05$ was considered statistically significant for all analyses.

\section{Results}

\section{Participants and dietary intakes}

All enrolled participants ( $n$ 20) completed the study in its entirety without any adverse effects (Fig. 1). Participants had fasting plasma glucose indicative of prediabetes (Table 2). On average, participants were considered obese on the basis of BMI, although five of the twenty participants had a BMI indicative of being overweight. They were also normolipidaemic, normotensive and not at increased risk for CVD based on established CIMT criteria ${ }^{(26)}$. According to HOMA-IR, values were indicative of insulin resistance based on established criteria $^{(32)}$. Participants' energy and nutrient intakes did not differ among trials with the exception that $\alpha$-tocopherol intakes were lower during GLU and WHITE compared with EGG and YOLK (online Supplementary Table S1). However, $\alpha$-tocopherol intakes were substantially lower than dietary recommendations regardless of trial, consistent with $92 \%$ of American men failing to meet dietary recommendations ${ }^{(33)}$.

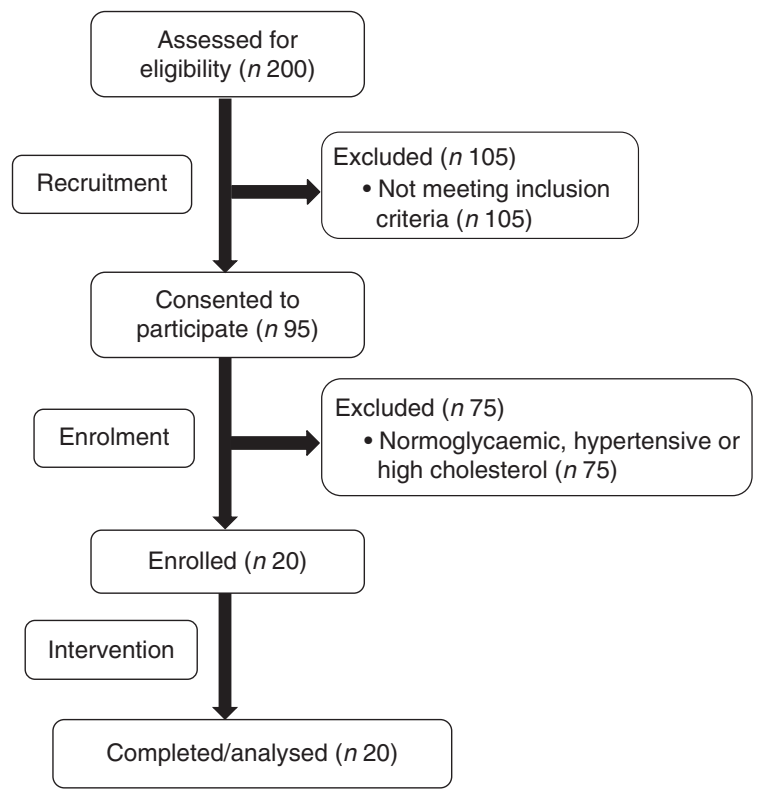

Fig. 1. Recruitment, enrolment, and intervention for prediabetic subjects who participated in the four-arm, randomised, cross-over trial.

\section{Brachial artery flow-mediated dilation}

Fasting FMD responses were not different $(P>0.05)$ among trials (Table 3). Postprandial changes in FMD ( $\triangle \mathrm{FMD}, \%)$ from baseline (i.e. $t=0 \mathrm{~min}$ ) showed main effects due to time, treatment, and time $\times$ treatment interaction effects $(P<0 \cdot 0001$; Fig. 2). FMD responses decreased relative to baseline at 30-60 min in GLU and 30-180 min in YOLK, but only at $30 \mathrm{~min}$ in EGG and WHITE. Compared with GLU and YOLK, decreases in FMD were attenuated at 30-60 min in EGG and WHITE, but YOLK was not different from GLU. FMD responses were lower in YOLK compared with WHITE at 90-180 min and EGG at 150-180 min. This was corroborated by $\mathrm{AUC}_{0-180 \text { min }}$ which was similarly higher in EGG and WHITE compared with GLU $(P<0.0001$; Fig. 2). Although FMD responses were lower in YOLK at 90-180 min compared with GLU, this was only significant at $150 \mathrm{~min}$ and did not result in a significant difference in $\mathrm{AUC}_{0-180 \mathrm{~min}}$. Blood pressure and heart rate did not differ among treatments at baseline or postprandially $(P>0.05$; data not shown). Pre-occlusion and maximal post-occlusion diameter and shear rate AUC did not differ among treatments ( $P>0.05$; Supplementary Table S2). These data suggest that co-ingestion of glucose with whole egg and egg white meals, but not yolks, protects against postprandial impairments in VEF.

\section{Plasma cholecystokinin, glucose, and insulin}

Fasting concentrations of plasma glucose, insulin, and CCK were not different among study visits (Table 3). Postprandial changes in CCK ( $\triangle \mathrm{CCK})$ showed treatment effects only. At $30 \mathrm{~min}$ and $120 \mathrm{~min}$ in WHITE and $90-180 \mathrm{~min}$ in EGG, CCK levels were greater compared with GLU $(P<0 \cdot 05$; Fig. 3(A)). Egg-mediated changes in CCK were corroborated by analysis of $\mathrm{AUC}_{0-180 \text { min }}(P<0 \cdot 01$; Fig. 3(A)) which was similarly higher in EGG and WHITE compared with GLU, whereas YOLK was not different from GLU or other egg-based meals.

Postprandial levels of $\Delta$ Glucose showed time and treatment effects (Fig. 3(B)). Specifically, plasma glucose increased at 30-120 min relative to baseline in GLU and YOLK but only at $30-90 \mathrm{~min}$ relative to baseline in EGG and WHITE.

Table 2. Participant characteristics

(Mean values with their standard errors; $n$ 20)

\begin{tabular}{lcc}
\hline & Mean & SEM \\
\hline Age (years) & 31.9 & 1.6 \\
BMI (kg/m²) & 31.1 & 0.7 \\
SBP (mmHg) & 123.0 & 6.0 \\
DBP (mmHg) & 72.0 & 4.0 \\
Plasma glucose (mmol/l) & 6.0 & 0.1 \\
Plasma insulin (pmol/l) & 144.8 & 15.8 \\
HOMA-IR & 5.3 & 0.6 \\
Plasma total cholesterol (mmol/l) & 5.0 & 0.1 \\
Plasma HDL-cholesterol (mmol/l) & 0.64 & 0.02 \\
Plasma LDL-cholesterol (mmol/l) & 3.7 & 0.1 \\
Plasma TAG (mmol/l) & 1.5 & 0.1 \\
Average left clMT (mm) & 0.56 & 0.04 \\
Average right clMT (mm) & 0.55 & 0.05 \\
\hline
\end{tabular}

SBP, systolic blood pressure; DBP, diastolic blood pressure; HOMA-IR, homoeostatic model assessment of insulin resistance; cIMT, carotid intima-media thickness. 
Table 3. Baseline values for flow-mediated dilation (FMD) and plasma biomarkers from each intervention arm (Mean values with their standard errors; $n$ 20)

\begin{tabular}{|c|c|c|c|c|c|c|c|c|c|}
\hline & \multicolumn{2}{|c|}{ GLU } & \multicolumn{2}{|c|}{ EGG } & \multicolumn{2}{|c|}{ WHITE } & \multicolumn{2}{|c|}{ YOLK } & \multirow[b]{2}{*}{$P$} \\
\hline & Mean & SEM & Mean & SEM & Mean & SEM & Mean & SEM & \\
\hline FMD (\%) & $7 \cdot 7$ & 0.4 & $7 \cdot 7$ & 0.4 & $7 \cdot 4$ & 0.4 & 8.0 & 0.4 & 0.68 \\
\hline CCK (pmol/l) & 13.5 & $0 \cdot 8$ & $12 \cdot 6$ & 0.7 & $13 \cdot 4$ & $0 \cdot 8$ & $13 \cdot 1$ & 0.9 & 0.47 \\
\hline Glucose $(\mathrm{mmol} / \mathrm{l})$ & 5.8 & 0.2 & $6 \cdot 1$ & 0.1 & $6 \cdot 1$ & 0.2 & $6 \cdot 0$ & 0.2 & 0.48 \\
\hline Insulin (pmol/l) & 134.5 & $30 \cdot 9$ & $164 \cdot 8$ & 35.9 & $130 \cdot 3$ & $28 \cdot 7$ & 149.4 & $32 \cdot 4$ & 0.77 \\
\hline Total cholesterol (mmol/l) & 4.9 & 0.2 & $5 \cdot 0$ & 0.2 & 4.9 & 0.2 & $5 \cdot 0$ & 0.2 & 0.86 \\
\hline HDL-cholesterol (mmol/l) & 0.7 & $0 \cdot 1$ & 0.6 & 0.1 & 0.7 & 0.1 & 0.7 & 0.1 & 0.13 \\
\hline LDL-cholesterol (mmol/l) & $3 \cdot 7$ & $0 \cdot 2$ & $3 \cdot 8$ & 0.2 & 3.7 & 0.2 & 3.6 & 0.2 & 0.87 \\
\hline TAG (mmol/l) & 1.3 & 0.1 & 1.5 & 0.2 & 1.6 & 0.2 & 1.5 & 0.2 & 0.54 \\
\hline MGO (nmol/l) & $76 \cdot 1$ & $3 \cdot 2$ & 73.9 & 3.4 & $72 \cdot 8$ & $2 \cdot 9$ & $72 \cdot 5$ & $3 \cdot 3$ & 0.41 \\
\hline 8-iso-PGF $2 a(\mathrm{pmol} / \mathrm{l})$ & 7638.0 & $1115 \cdot 0$ & $7924 \cdot 0$ & 1271.0 & $7232 \cdot 0$ & 939.0 & $7087 \cdot 0$ & 859.0 & 0.75 \\
\hline AA $(\mu \mathrm{mol} / \mathrm{l})$ & 755.0 & $32 \cdot 0$ & $725 \cdot 0$ & 34.0 & $749 \cdot 0$ & $32 \cdot 0$ & $750 \cdot 0$ & 34.0 & 0.32 \\
\hline
\end{tabular}

GLU, glucose; EGG, whole eggs; WHITE, egg whites; YOLK, egg yolks; CCK, cholecystokinin; MGO, methylglyoxal; 8-iso-PGF $2 a$, 8-isoprostaglandin- $\mathrm{F}_{2 a}$; $A A$, arachidonic acid.
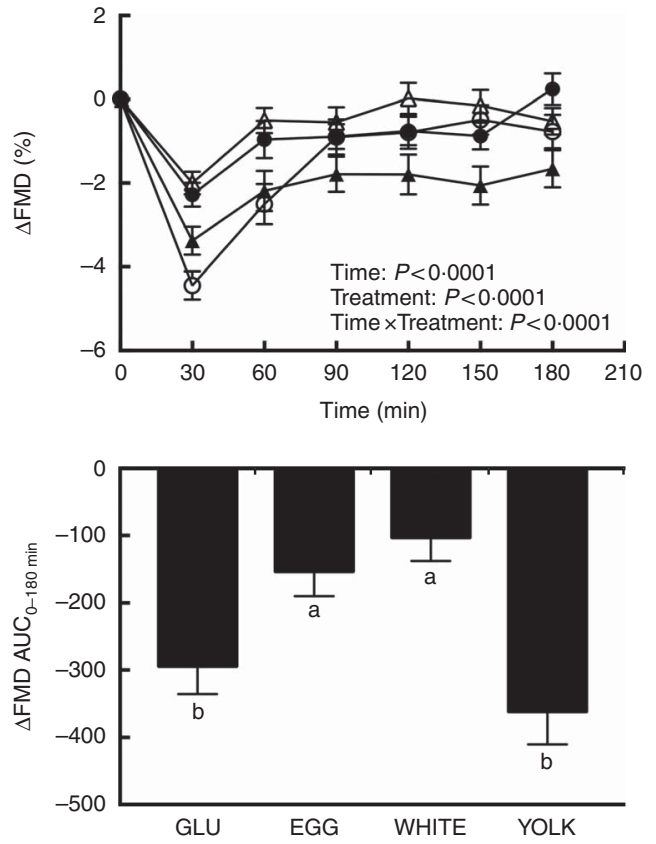

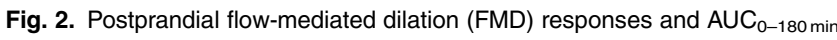
following ingestion of glucose in the absence or presence of egg-based meals by prediabetic men. Postprandial responses were analysed using two-way repeated-measures (RM) ANOVA with Bonferroni's post hoc test. $\mathrm{AUC}_{0-180 \mathrm{~min}}$ was calculated using the trapezoidal rule and analysed using one-way RM ANOVA with Bonferroni's post hoc test. Values are means ( $n$ 20), with their standard errors. $\_$, Glucose (GLU); - - , whole eggs (EGG); $\triangle$, egg whites (WHITE); $\_$, egg yolk (YOLK). ${ }^{\text {a,b }}$ Mean values with unlike letters are significantly different $(P<0.05)$.

Furthermore, increases were significantly attenuated at $120 \mathrm{~min}$ in EGG and WHITE relative to GLU, whereas YOLK was not different from GLU. AUC ${ }_{0-180 \text { min }}$ of $\Delta$ Glucose showed that EGG and WHITE were similarly lowered compared with GLU whereas YOLK was not different from GLU, EGG or WHITE.

$\Delta$ Insulin showed time, treatment and time $\times$ treatment interactive effects (Fig. 3(C)) such that insulin increased relative to baseline at the following times in each treatment: GLU (30-150 min), EGG (30-120 min), WHITE (30-90 min), YOLK (30-120 min). Compared with GLU, insulin increased to a lesser extent in EGG and WHITE at 90-150 min. In addition, insulin increased to a greater extent at $30 \mathrm{~min}$ in WHITE compared with EGG. $A C_{0-180 \text { min }}$ of postprandial insulinaemia showed similarly lower levels in EGG and WHITE compared with GLU, whereas YOLK was not different from GLU or other egg-based meals. In addition, $\Delta \mathrm{FMD}_{\mathrm{AUC}}$ and $\Delta \mathrm{Insulin}_{\mathrm{AUC}}$ were negatively correlated $(r-0 \cdot 28, P<0 \cdot 05)$, suggesting that lowering of insulinaemia is associated with improved VEF. Collectively, these data suggest that vasoprotection in EGG and WHITE is likely due to attenuating glycaemia and insulinaemia, which may be attributed to a CCK-mediated delay in gastric emptying.

\section{Plasma lipids}

Fasting concentrations of plasma TAG and total, HDL and LDLcholesterol did not differ among study trials (Table 3) nor did their respective postprandial $\mathrm{AUC}_{0-180 \text { min }}$ responses differ among treatments (Fig. 4). However, there were main effects due to time (Fig. 4(A)-(D)). Plasma TAG showed a significant main effect of time. However, this occurred without any statistically significant post hoc effects for time. Plasma total cholesterol decreased postprandially and failed to return to baseline levels by $180 \mathrm{~min}$. Plasma LDL-cholesterol followed the same trend as total cholesterol. Plasma HDL-cholesterol decreased at 60-150 min relative to baseline before returning to baseline by $180 \mathrm{~min}$.

\section{Plasma methylglyoxal}

Fasting MGO concentrations did not differ among trials (Table 3). Plasma $\Delta \mathrm{MGO}$ responses were unaffected by treatment, consistent with no difference in $\mathrm{AUC}_{0-180 \text { min }}$ between treatments despite EGG and WHITE being 37-45\% lower than GLU (Fig. 5). However, MGO response did exhibit a statistically significant main effect for time. Specifically, plasma MGO levels were increased at 30-120 min postprandially relative to baseline before returning to levels no different from baseline by $150 \mathrm{~min}$. MGO levels peaked at $30 \mathrm{~min}$, which was greater $(P<0.05)$ than levels at 90-180 min. Additionally, MGO levels were greater at $60 \mathrm{~min}$ relative to $90-180 \mathrm{~min}$, at $90 \mathrm{~min}$ relative to $120-150 \mathrm{~min}$ and at $120 \mathrm{~min}$ relative to $150-180 \mathrm{~min}$. 

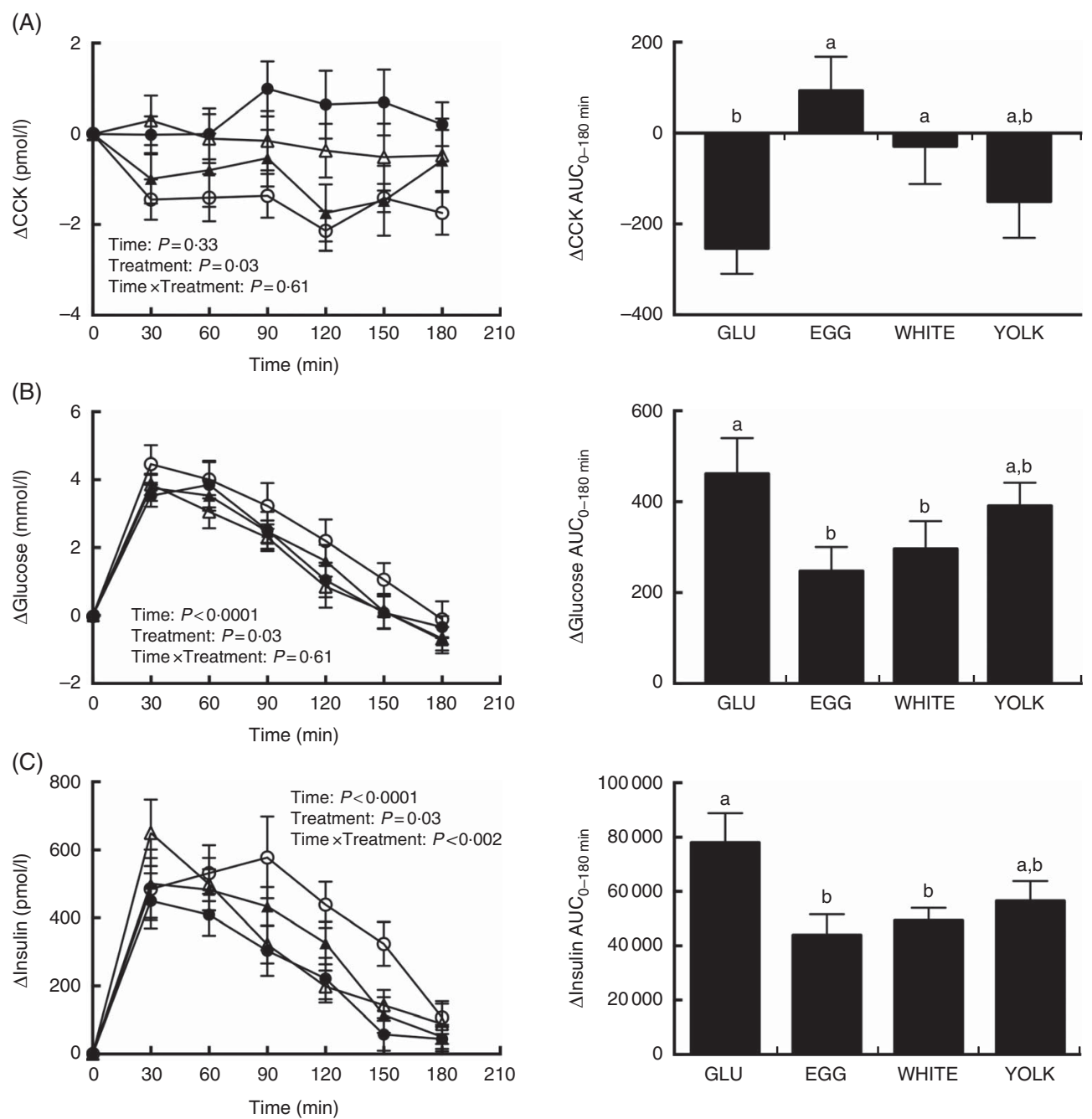

Fig. 3. Postprandial cholecystokinin (CCK) (A) glucose (B) and insulin (C) responses and $A_{U} \mathrm{C}_{0-180 \text { min }}$ following ingestion of glucose in the absence or presence of egg-based meals by prediabetic men. Postprandial responses were analysed using two-way repeated-measures (RM) ANOVA with Bonferroni's post hoc test. AUC $_{0-180 \mathrm{~min}}$ was calculated using the trapezoidal rule and analysed using one-way RM ANOVA with Bonferroni's post hoc test. Values are means $(n$ 20), with their standard errors. $-\_$, Glucose (GLU); $-\subset$, whole eggs (EGG); $\_$, egg whites (WHITE); $\_$, egg yolk (YOLK). ${ }^{\text {a,b }}$ Mean values with unlike letters are significantly different $(P<0.05)$.

\section{Plasma $F_{2}$-isoprostanes and arachidonic acid}

Baseline concentrations of 8-iso- $\mathrm{PGF}_{2 \alpha}$ and $\mathrm{AA}$ did not differ among trials (Table 3). Main effects due to time and treatment were observed for postprandial changes in plasma 8-iso-PGF $2 \alpha$ (Fig. 6(A)). Relative to baseline, $\Delta 8-i s o-\mathrm{PGF}_{2 \alpha}$ levels increased at 60-150 $\mathrm{min}$ in GLU, $120 \mathrm{~min}$ in EGG, 90-120 min in WHITE, and $30-150 \mathrm{~min}$ in YOLK. In addition, compared with GLU, increases were attenuated at 90-120 min in WHITE and 90-150 min in EGG, whereas YOLK did not differ from GLU at any time point. Although we observed treatment effects at the aforementioned time points postprandially, we did not observe any differences in AUC despite 39-54\% lower values in EGG and WHITE compared with GLU. Because $\mathrm{F}_{2}$-IsoPs are derived from AA, we calculated the ratio of $8-i s o-\mathrm{PGF}_{2 \alpha}$ :AA as an indicator of the extent of lipid peroxidation. $\triangle \mathrm{AA}$ decreased postprandially regardless of treatment $(P<0 \cdot 01)$, with levels significantly lower at 90-180 min compared with baseline
(Fig. 6(B)). We therefore normalised changes in 8-iso-PGF ${ }_{2 \alpha}$ to changes in AA (Fig. 6(C)). Data showed significant time, treatment and time $\times$ treatment interactive effects in $\Delta 8$-iso-PGF $\mathrm{PG}_{2 \alpha}$ : $\Delta \mathrm{AA}$. Relative to baseline, the ratio of $8-i s o-\mathrm{PGF}_{2 \alpha}: \mathrm{AA}$ increased at 60-180 $\mathrm{min}$ in GLU, 90-120 $\mathrm{min}$ in EGG, 90-150 $\mathrm{min}$ in WHITE and $30-180 \mathrm{~min}$ in YOLK. In addition, 8-iso-PGF $2 \alpha$ :AA was increased to a lesser extent at 90-180 min in EGG and WHITE compared with GLU, with YOLK not different from GLU. Likewise, $\mathrm{AUC}_{0-180 \text { min }}$ was similarly lower in EGG and WHITE compared with GLU, whereas YOLK was not different from GLU and higher than EGG and WHITE. This finding suggests egg- and egg white-based meals protect against PPH-mediated impairments in VEF by attenuating lipid peroxidation.

\section{Discussion}

This study demonstrates that co-ingestion of whole eggs with glucose protects against PPH-mediated impairments in VEF by 

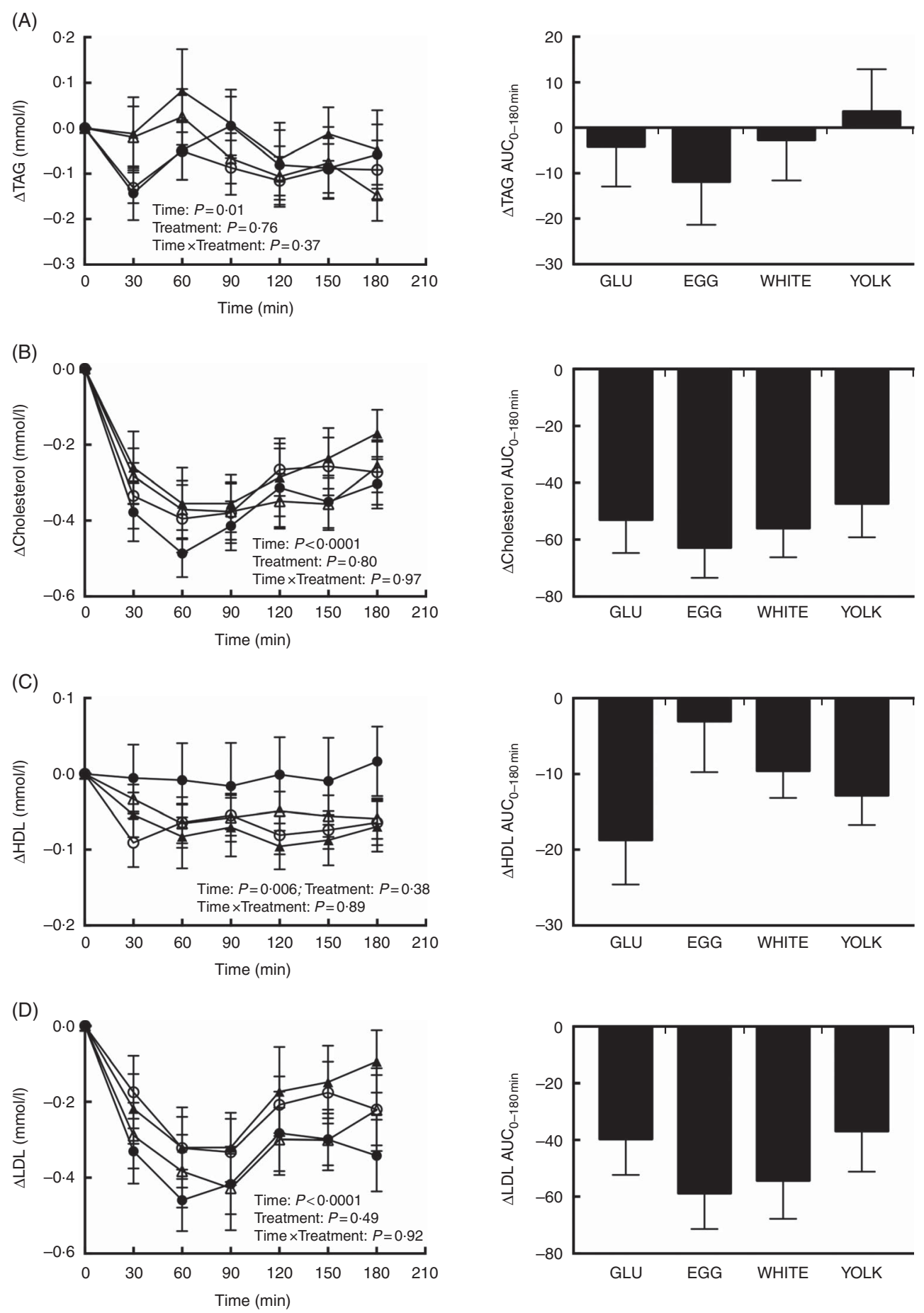

Fig. 4. Postprandial TAG (A), total cholesterol (B), HDL-cholesterol (C) and LDL-cholesterol (D) responses and $A U C_{0-180 \text { min }}$ following ingestion of glucose in the absence or presence of egg-based meals by prediabetic men. Postprandial responses were analysed using two-way repeated-measures (RM) ANOVA with Bonferroni's post hoc test. $\mathrm{AUC}_{0-180 \mathrm{~min}}$ was calculated using the trapezoidal rule and analysed using one-way RM ANOVA with Bonferroni's post hoc test. Values are

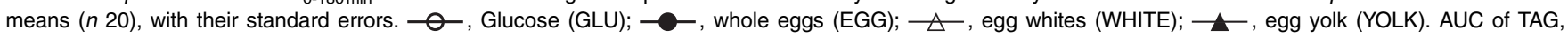
cholesterol, HDL and LDL did not differ among treatments $(P>0.05)$.

limiting lipid peroxidation independent of changes in MGO or circulating lipids. This is potentially mediated by the egg white fraction, based on whole egg and egg white meals similarly attenuating glycaemia and lipid peroxidation. Vasoprotective activities of whole eggs and egg whites occurred in association with gut-level improvements in CCK, which would be expected to limit PPH-mediated lipid peroxidation by delaying gastric emptying. Thus, simply reducing the glucose content in eggbased meals did not confer vasoprotection, but rather replacing carbohydrate with either whole eggs or egg whites limits postprandial impairments in VEF otherwise induced by glucose ingestion. 

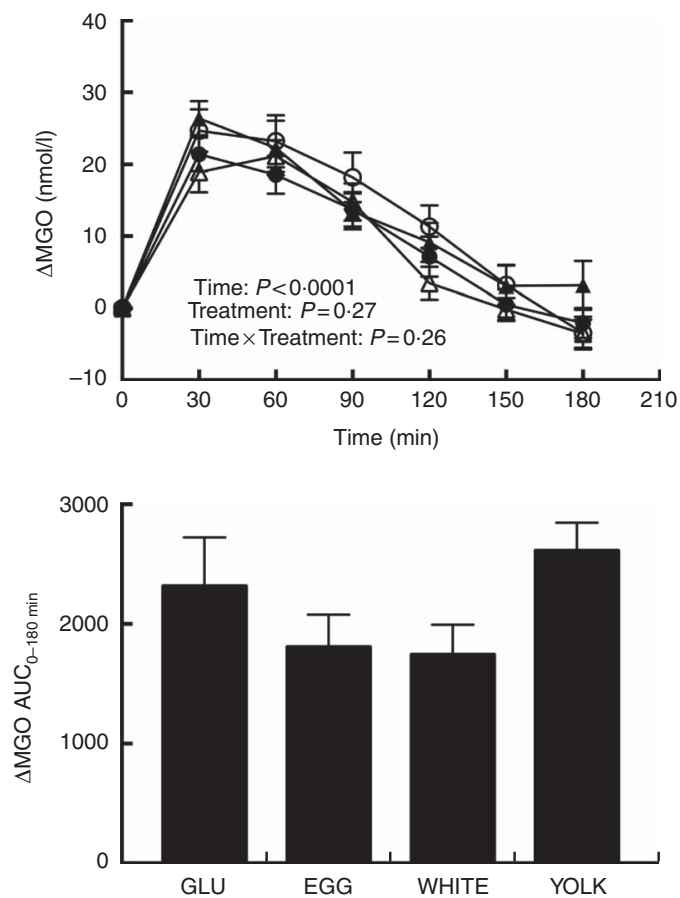

Fig. 5. Postprandial methylglyoxal (MGO) responses and $A \cup C_{0-180 m i n}$ following ingestion of glucose in the absence or presence of egg-based meals by prediabetic men. Postprandial responses were analysed using twoway repeated-measures (RM) ANOVA with Bonferroni's post hoc test. $\mathrm{AUC}_{0-180 \mathrm{~min}}$ was calculated using the trapezoidal rule and analysed using one-way RM ANOVA with Bonferroni's post hoc test. Values are means ( $n 20)$, with their standard errors. $-\subset$, Glucose (GLU); -- , whole eggs (EGG); $\triangle$, egg whites (WHITE); $\_$, egg yolk (YOLK). MGO AUC did not differ among treatments $(P>0.05)$.

Test meals were formulated to be isoenergetic rather than equal in carbohydrate. This approach was undertaken specifically to rule out confounding effects of varying energy intakes on postprandial responses. This is consistent with others showing that carbohydrate quantity in test meals that varied in energy content differentially affected postprandial glycaemia ${ }^{(34)}$. In addition, the use of egg-based test meals served as a modifiable food matrix to investigate the influence of the macronutrient ratio on postprandial responses. Findings show an inter-related role between the intestinal hormone CCK, PPH, and downstream lipid peroxidation, suggesting that whole egg and egg white-based meals mediate vasoprotection along the gut-vessel axis. Data show that co-ingestion of glucose with whole eggs and egg whites, but not egg yolks, attenuates postprandial decreases in FMD otherwise lowered by glucose ingestion alone. Reducing glucose quantity did not improve FMD responses, but rather the macronutrient being substituted in lieu of glucose played an important role. Indeed, meals containing the egg white fraction attenuated postprandial impairments in VEF as assessed by FMD, suggesting that eggfraction composition differences in meals regulate VEF. Future studies are warranted to examine dose-response effects of egg whites and yolks in relation to whole eggs on postprandial vascular health.

Bioactive peptides resulting from enzymatic hydrolysis of egg white and yolk proteins exhibit blood pressure-lowering activities in spontaneously hypertensive rats $(\mathrm{SHR})^{(35,36)}$. However, feeding egg white hydrolysates in SHR compared with egg yolk hydrolysates resulted in a greater lowering of blood pressure ${ }^{(37)}$. Contrary to those findings, blood pressure was unaffected by egg treatments in our study, and likely reflects participants' normotensive status. Nonetheless, mesenteric arteries of SHR treated with whole egg hydrolysates ${ }^{(37)}$ or the egg white-derived peptide ovokinin ${ }^{(38)}$ exhibited greater $\mathrm{NO}^{\circ}$-dependent vasorelaxation consistent with our findings of egg-mediated improvements in FMD responses that reflect $\mathrm{NO}^{\bullet}$-dependent vasodilation ${ }^{(12)}$.

In hyperlipidaemic adults, 6 weeks daily consumption of 0.5 cups egg substitute ( $99 \%$ egg whites), but not an equal amount of protein from whole eggs, improved fasting FMD responses ${ }^{(21)}$. To the contrary, we show improvements in FMD following consumption of either whole eggs or egg whites. Differences are likely due to study duration in which our postprandial study had strict dietary control. We also acknowledge that differences in protein quantity may also explain vasoprotection by WHITE (25 g) and EGG (9.5 g) compared with YOLK (5g). No postprandial studies have examined the dose-dependent effect of egg protein on glycaemia and FMD. However, postprandial studies demonstrate that glycaemic responses were dose-dependently attenuated by increasing amounts of whey protein isolate that was co-ingested with glucose ${ }^{(39)}$. Thus, future studies are needed to determine differential and dose-dependent effects of egg white and egg yolk proteins with a carbohydrate challenge on postprandial glycaemia and FMD.

Gastric emptying helps to regulate postprandial glycaemia, indicating a role of gut physiology on postprandial VEF. CCK functions to delay gastric emptying and its secretion is greatest in response to dietary protein ${ }^{(40)}$. In agreement, we showed that CCK was greatest in response to meals containing whole eggs and egg whites. This is consistent with others showing greater CCK following the co-ingestion of whole eggs with a standardised mixed-meal compared with the mixed meal alone ${ }^{(22)}$. This may be attributed to whole egg hydrolysates increasing CCK in murine endocrine cells ${ }^{(41)}$ and egg white peptides inhibiting intestinal proteases ${ }^{(42)}$ that would otherwise digest intraluminal CCK releasing factors leading to lower levels of $\mathrm{CCK}^{(43)}$. Contrary to these findings, we showed greater CCK levels following consumption of the mixed-meal with egg whites but not egg yolks. These differences may reflect the higher energy content of test meals and/or varying quantity of egg components (i.e. two whites $v$. two yolks) used in earlier studies $^{(22)}$. Differences in participant health status may also account for observed changes in that we studied prediabetic, obese adults rather than healthy adults ${ }^{(22)}$. Indeed, obese mice fed a high-fat diet had lower small intestinal CCK expression ${ }^{(44)}$. Obese adults also exhibit greater CCK following a high-protein meal compared with a high-fat meal ${ }^{(40)}$. Although increasing the solid mass content of a solid/liquid meal delays gastric emptying $^{(45)}$, which would be expected to be reflected by greater levels of CCK, we did not observe any increases in CCK based on increasing solid food mass in egg-based meals. Future studies are needed to examine egg white-derived bioactive peptides on other gut hormones (e.g. PYY, GLP-1) that also regulate gastric emptying. 
(A)
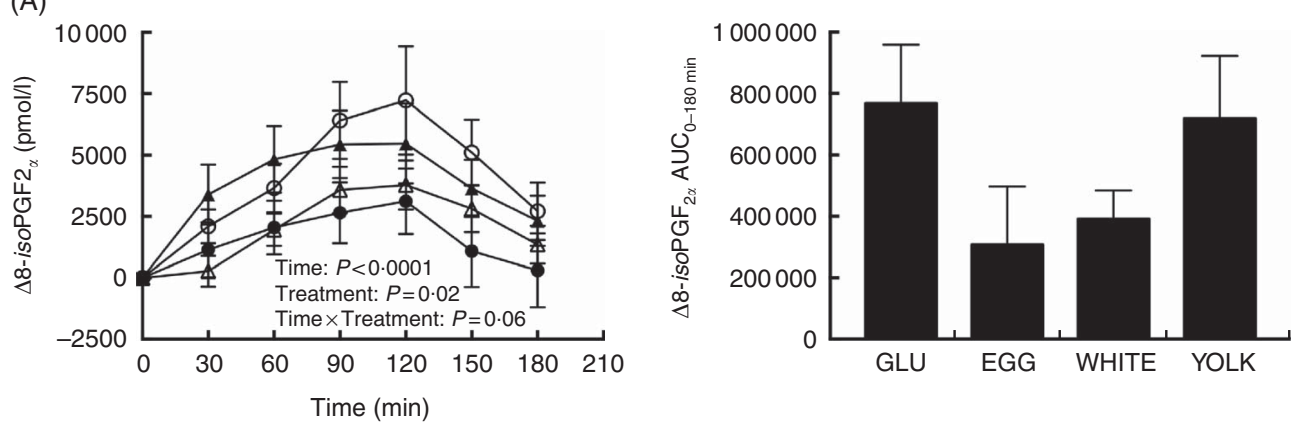

(B)
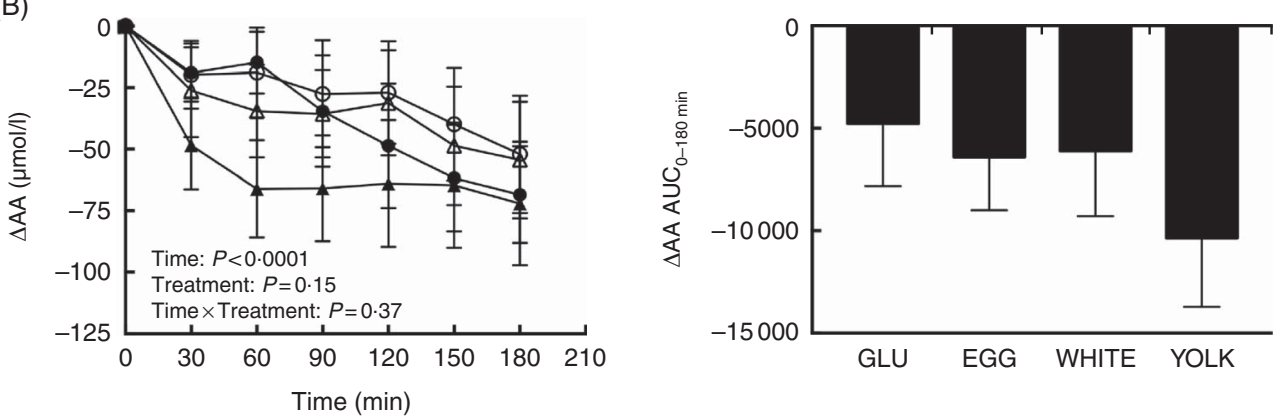

(C)
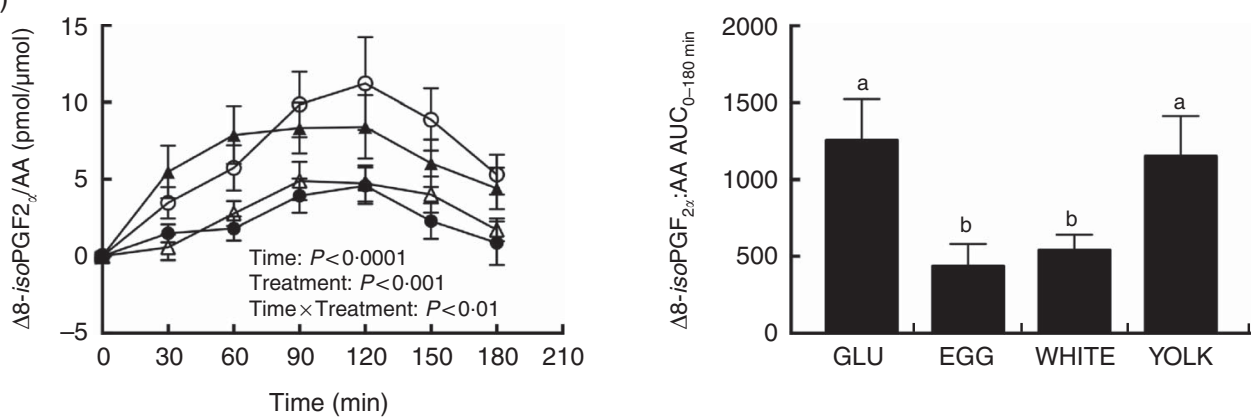

Fig. 6. Postprandial responses and $A \mathrm{UC}_{0-180 \mathrm{~min}}$ for 8-isoprostaglandin $\mathrm{F}_{2 a}\left(8-i s o-\mathrm{PGF}_{2 a}\right)(\mathrm{A})$, arachidonic acid (AA) (B), and the ratio of 8-iso-PGF ${ }_{2 a}: A A(C)$ following ingestion of glucose in the absence or presence of egg-based meals by prediabetic men. Postprandial responses were analysed using two-way repeated-measures (RM) ANOVA with Bonferroni's post hoc test. AUC ${ }_{0-180 \text { min }}$ was calculated using the trapezoidal rule and analysed using one-way RM ANOVA with Bonferroni's post hoc test. Values are means $(n 20)$, with their standard errors. $\_$, Glucose (GLU); ——, whole eggs (EGG); $₫$, egg whites (WHITE); $\longrightarrow$, egg yolk (YOLK). a,b Mean values with unlike letter are significantly different $(P<0.05)$.

Whole egg and egg white meals attenuated postprandial glycaemia compared with glucose ingestion alone, which is expected due to decreased carbohydrate quantity. However, glycaemic responses following ingestion of the egg yolk meal was not different compared with glucose ingestion alone, demonstrating that simply reducing carbohydrate content did not lead to an attenuation of glycaemia. Whole eggs as part of a standard breakfast limited postprandial glycaemia in healthy adults $^{(22)}$. Chronic ingestion of 2 eggs/d as part of a highprotein diet for 12 weeks also improved 2-h blood glucose following a glucose challenge in adults with the metabolic syndrome (MetS) ${ }^{(18)}$. Inclusion of protein with a glucose challenge also attenuated postprandial glycaemia, likely by delaying gastric emptying, as protein is a potent stimuli for gastric regulatory hormones ${ }^{(46)}$. We observed that peak insulin concentrations $(30 \mathrm{~min})$ were greater in WHITE compared with EGG, which may have aided in glucose uptake. Whole egg and egg white, but not egg yolk meals, attenuated postprandial insulinaemia compared with glucose ingestion alone. Insulin binding to its receptor results in phosphorylation of its substrate (IRS-1). This leads to the activation of protein kinase B (Akt), which phosphorylates eNOS at Ser ${ }^{1177}$ and increases its activity to stimulate $\mathrm{NO}^{\bullet}$ production ${ }^{(47)}$. However, despite increased insulin concentrations during GLU compared with EGG and WHITE, we observed that postprandial FMD responses declined. This may be due to oxidative stress impairing insulin signalling ${ }^{(47)}$, but future studies are needed to determine any protective effect of egg whites and yolks on oxidative-stress mediated impairments in insulin signalling.

Glucose induces oxidative stress through increased formation of mitochondrial superoxide, accumulation of proinflammatory mediators and advanced glycation end products, and activity of enzymes (e.g. protein kinase C, NADPH oxidase) that contribute to the generation of reactive oxygen species $(\mathrm{ROS})^{(3,4,10)}$. Regardless of source, glucose-induced increases in ROS decrease NO bioavailability thereby leading to vascular dysfunction ${ }^{(4,48)}$. 
PPH has been shown to increase $\mathrm{MGO}^{(30)}$. MGO also increases mitochondrial ROS generation ${ }^{(49)}$, and increases in MGO following glucose ingestion impairs VEF as assessed by FMD in $\operatorname{dog} s^{(50)}$. However, in this study, improvements in VEF were independent of changes in MGO as responses did not differ between test meals. In addition, improvements in VEF occurred independent of changes in plasma lipids. Thus, we measured $\mathrm{F}_{2}$-IsoPs/AA to examine $\mathrm{PPH}$-mediated lipid peroxidation on $\mathrm{VEF}^{(9)}$. We provide novel evidence that co-ingestion of glucose with whole eggs or egg whites attenuated increases in $\mathrm{F}_{2}$-IsoPs/ AA. This suggests that the vasoprotective activities of whole eggs and eggs whites are due to limiting glycaemia and downstream lipid peroxidation, consistent with evidence that oxidative stress is positively correlated with the magnitude of $\mathrm{PPH}$ and that $\mathrm{PPH}$ and lipid peroxidation are inversely related with $\mathrm{FMD}^{(5,13)}$. In diabetic adults, $\mathrm{F}_{2}$-IsoPs increased following a glucose challenge ${ }^{(7)}$ and both whole eggs and a yolk-free substitute lowered plasma oxidised LDL in the MetS adults ${ }^{(18)}$. Although hydrolysates from egg whites and yolks exhibit free-radical scavenging activity ${ }^{(51,52)}$, we show that egg yolk meals failed to attenuate lipid peroxidation, suggesting a more prominent role of bioactive constituents of egg whites to improve VEF.

Separate from serving as a marker of oxidative stress, $\mathrm{F}_{2}$-IsoPs provoke CVD by modulating platelet activation, vascular inflammation, inducing vasoconstriction and vascular smooth muscle cell proliferation, inhibiting angiogenesis, and dysregulating cardiac ion channels ${ }^{(9)}$. Furthermore, AA can be enzymatically (i.e. cyclooxygenase, lipoxygenase) oxidised to prostaglandins, thromboxanes, and leukotrienes, which exert similar action as $\mathrm{F}_{2}$-IsoPs ${ }^{(53)}$. The physiologic roles of $\mathrm{F}_{2}$-IsoPs and AA metabolites to negatively affect VEF occur separate from directly reducing $\mathrm{NO}^{\bullet}$ bioavailability (i.e. $\mathrm{NO}^{\bullet}$-independent). Thus, more research is needed to examine $\mathrm{NO}^{\circ}$-dependent and -independent pathways on VEF in response to egg-based meals. Prior postprandial studies in adults with the MetS suggest no sex-specific effects ${ }^{(13)}$, therefore women were not included, but future studies should examine the effects of sex hormones on responses to egg-based meals.

In conclusion, this study shows that replacing a portion of an oral glucose challenge with whole eggs or egg whites, but not yolks, protects against postprandial decreases in VEF by attenuating glycaemia and oxidative stress. Co-ingestion of carbohydrate-based meals with whole eggs or egg whites may serve as an effective dietary approach to mitigate PPH-mediated impairments in VEF that otherwise increases CVD risk. However, future studies are needed to examine the vasoprotective benefits of eggs as part of more complex mixed meals. Nonetheless, in the absence of validated dietary strategies, the accumulation of acute insults mediated by $\mathrm{PPH}$ at the vascular endothelium that contribute to CVD risk will remain problematic. This is of public health importance as half of adult Americans have prediabetes or diabetes ${ }^{(54)}$, and humans spend the majority of their day in the postprandial state.

\section{Acknowledgements}

The authors thank Kevin Schill for their assistance with J. D. M. in coordinating the study and analysis of vascular data.
The present study was funded by the American Egg Board/ Egg Nutrition Center. American Egg Board/Egg Nutrition Center had no role in the design, analysis, or writing of this article.

The authors contributions are as follows: R. S. B., J. S. V. and E. M. were responsible for the study design; A. N. L. analysed participant dietary records. J. D. M., C. C., J. L., E. J. R. and K. D. B. were responsible for collecting and analysing data; and J. D. M. and R. S. B. wrote the initial draft of the manuscript and all authors contributed to the editing and review of this manuscript. All authors read and approved the final manuscript.

The authors declare that there are no conflicts of interest.

\section{Supplementary material}

For supplementary material/s referred to in this article, please visit https://doi.org/10.1017/S0007114517003610

\section{References}

1. Mozaffarian D, Benjamin EJ, Go AS, et al. (2016) Heart disease and stroke statistics-2016 update: a report from the American Heart Association. Circulation 133, e38-e360.

2. DECODE Study Group; on behalf of the European Diabetes Epidemiology Group (2001) Glucose tolerance and cardiovascular mortality: comparison of fasting and 2-hour diagnostic criteria. Arch Intern Med 161, 397-405.

3. Jacome-Sosa M, Parks EJ, Bruno RS, et al. (2016) Postprandial metabolism of macronutrients and cardiometabolic risk: recent developments, emerging concepts, and future directions. Adv Nutr 7, 364-374.

4. Mah E \& Bruno RS (2012) Postprandial hyperglycemia on vascular endothelial function: mechanisms and consequences. Nutr Res 32, 727-740.

5. Mah E, Noh SK, Ballard KD, et al. (2011) Postprandial hyperglycemia impairs vascular endothelial function in healthy men by inducing lipid peroxidation and increasing asymmetric dimethylarginine:arginine. $J$ Nutr $\mathbf{1 4 1}$, 1961-1968.

6. Liu VW \& Huang PL (2008) Cardiovascular roles of nitric oxide: a review of insights from nitric oxide synthase gene disrupted mice. Cardiovasc Res 77, 19-29.

7. Sampson MJ, Gopaul N, Davies IR, et al. (2002) Plasma F2 isoprostanes: direct evidence of increased free radical damage during acute hyperglycemia in type 2 diabetes. Diabetes Care $\mathbf{2 5}$, 537-541.

8. Ceriello A, Esposito K, Piconi L, et al. (2008) Oscillating glucose is more deleterious to endothelial function and oxidative stress than mean glucose in normal and type 2 diabetic patients. Diabetes 57, 1349-1354.

9. Bauer J, Ripperger A, Frantz S, et al. (2014) Pathophysiology of isoprostanes in the cardiovascular system: implications of isoprostane-mediated thromboxane A2 receptor activation. Br J Pharmacol 171, 3115-3131.

10. Giacco F \& Brownlee M (2010) Oxidative stress and diabetic complications. Circ Res 107, 1058-1070.

11. Matsuzawa Y, Kwon TG, Lennon RJ, et al. (2015) Prognostic value of flow-mediated Vasodilation in brachial artery and fingertip artery for cardiovascular events: a systematic review and meta-analysis. J Am Heart Assoc 4, 11.

12. Harris RA, Nishiyama SK, Wray DW, et al. (2010) Ultrasound assessment of flow-mediated dilation. Hypertension $\mathbf{5 5}$, $1075-1085$ 
13. Ballard KD, Mah E, Guo Y, et al. (2013) Low-fat milk ingestion prevents postprandial hyperglycemia-mediated impairments in vascular endothelial function in obese individuals with metabolic syndrome. J Nutr 143, 1602-1610.

14. Djoussé L \& Gaziano JM (2008) Egg consumption in relation to cardiovascular disease and mortality: the Physicians' Health Study. Am J Clin Nutr 87, 964-969.

15. Fuller NR, Sainsbury A, Caterson ID, et al. (2015) Egg consumption and human cardio-metabolic health in people with and without diabetes. Nutrients 7, 7399-7420.

16. Li Y, Zhou C, Zhou X, et al. (2013) Egg consumption and risk of cardiovascular diseases and diabetes: a meta-analysis. Atherosclerosis 229, 524-530.

17. Rong Y, Chen L, Zhu T, et al. (2013) Egg consumption and risk of coronary heart disease and stroke: dose-response metaanalysis of prospective cohort studies. BMJ 346, e8539.

18. Blesso CN, Andersen CJ, Barona J, et al. (2013) Whole egg consumption improves lipoprotein profiles and insulin sensitivity to a greater extent than yolk-free egg substitute in individuals with metabolic syndrome. Metabolism 62, 400-410.

19. Katz DL, Evans MA, Nawaz H, et al. (2005) Egg consumption and endothelial function: a randomized controlled crossover trial. Int J Cardiol 99, 65-70.

20. Katz DL, Gnanaraj J, Treu JA, et al. (2015) Effects of egg ingestion on endothelial function in adults with coronary artery disease: a randomized, controlled, crossover trial. Am Heart J 169, 162-169.

21. Njike V, Faridi Z, Dutta S, et al. (2010) Daily egg consumption in hyperlipidemic adults - effects on endothelial function and cardiovascular risk. Nutr J 9, 1-9.

22. Pelletier X, Thouvenot P, Belbraouet S, et al. (1996) Effect of egg consumption in healthy volunteers: influence of yolk, white or whole-egg on gastric emptying and on glycemic and hormonal responses. Ann Nutr Metab 40, 109-115.

23. Garces-Rimon M, Gonzalez C, Uranga JA, et al. (2016) Pepsin egg white hydrolysate ameliorates obesity-related oxidative stress, inflammation and steatosis in zucker fatty rats. PLOS ONE 11, e0151193.

24. American Diabetes Association (2011) Standards of medical care in diabetes - 2011. Diabetes Care 34, S11-S61.

25. U.S. Department of Agriculture Agricultural Research Service (2016) Nutrient Intakes from Food and Beverages: Mean Amounts Consumed per Individual, by Gender and Age, What We Eat in America, NHANES 2013-2014. Beltsville, MD: U.S. Department of Agriculture Agricultural Research Service.

26. Stein JH, Korcarz CE, Hurst RT, et al. (2008) Use of carotid ultrasound to identify subclinical vascular disease and evaluate cardiovascular disease risk: a consensus statement from the American Society of Echocardiography Carotid IntimaMedia Thickness Task Force. Endorsed by the Society for Vascular Medicine. J Am Soc Echocardiogr 21, 93-111; quiz 189-190.

27. Friedewald WT, Levy RI \& Fredrickson DS (1972) Estimation of the concentration of low-density lipoprotein cholesterol in plasma, without use of the preparative ultracentrifuge. Clin Chem 18, 499-502.

28. Matthews DR, Hosker JP, Rudenski AS, et al. (1985) Homeostasis model assessment: insulin resistance and beta-cell function from fasting plasma glucose and insulin concentrations in man. Diabetologia 28, 412-419.

29. Taylor AW, Bruno RS, Frei B, et al. (2006) Benefits of prolonged gradient separation for high-performance liquid chromatography-tandem mass spectrometry quantitation of plasma total 15-series F-isoprostanes. Anal Biochem 350 , $41-51$.
30. Masterjohn C, Mah E, Guo Y, et al. (2012) gamma-Tocopherol abolishes postprandial increases in plasma methylglyoxal following an oral dose of glucose in healthy, collegeaged men. J Nutr Biochem 23, 292-298.

31. Bland JM \& Altman DG (1995) Calculating correlation coefficients with repeated observations: part 1 - correlation within subjects. BMJ 310, 446.

32. Gayoso-Diz P, Otero-González A, Rodriguez-Alvarez MX, et al. (2013) Insulin resistance (HOMA-IR) cut-off values and the metabolic syndrome in a general adult population: effect of gender and age: EPIRCE cross-sectional study. BMC Endocrine Disorders 13, 47-57.

33. Maras JE, Bermudez OI, Qiao N, et al. (2004) Intake of alphatocopherol is limited among US adults. J Am Diet Assoc 104, 567-575.

34. Wolever TM \& Bolognesi C (1996) Prediction of glucose and insulin responses of normal subjects after consuming mixed meals varying in energy, protein, fat, carbohydrate and glycemic index. J Nutr 126, 2807-2812.

35. Miguel M, Lopez-Fandino R, Ramos M, et al. (2005) Short-term effect of egg-white hydrolysate products on the arterial blood pressure of hypertensive rats. BrJ Nutr $\mathbf{9 4}$, 731-737.

36. Yoshii H, Tachi N, Ohba R, et al. (2001) Antihypertensive effect of ACE inhibitory oligopeptides from chicken egg yolks. Comp Biochem Physiol C Toxicol Pharmacol 128, $27-33$.

37. Jahandideh F, Majumder K, Chakrabarti S, et al. (2014) Beneficial effects of simulated gastro-intestinal digests of fried egg and its fractions on blood pressure, plasma lipids and oxidative stress in spontaneously hypertensive rats. PLOS ONE 9, e115006.

38. Matoba N, Usui H, Fujita H, et al. (1999) A novel antihypertensive peptide derived from ovalbumin induces nitric oxide-mediated vasorelaxation in an isolated SHR mesenteric artery. FEBS Lett 452, 181-184.

39. Paterson MA, Smart CEM, Lopez PE, et al. (2017) Increasing the protein quantity in a meal results in dose-dependent effects on postprandial glucose levels in individuals with type 1 diabetes mellitus. Diabet Med 34, 851-854.

40. Brennan IM, Luscombe-Marsh ND, Seimon RV, et al. (2012) Effects of fat, protein, and carbohydrate and protein load on appetite, plasma cholecystokinin, peptide YY, and ghrelin, and energy intake in lean and obese men. Am J Physiol Gastrointest Liver Physiol 303, G129-G140.

41. Geraedts MC, Troost FJ, Fischer MA, et al. (2011) Direct induction of CCK and GLP-1 release from murine endocrine cells by intact dietary proteins. Mol Nutr Food Res 55, 476-484.

42. Kovacs-Nolan J, Phillips M \& Mine Y (2005) Advances in the value of eggs and egg components for human health. J Agric Food Chem 53, 8421-8431.

43. Liddle RA (1995) Regulation of cholecystokinin secretion by intraluminal releasing factors. $A m J$ Physiol 269, G319-G327.

44. Duca FA, Swartz TD, Sakar Y, et al. (2013) Decreased intestinal nutrient response in diet-induced obese rats: role of gut peptides and nutrient receptors. Int J Obes 37, 375-381.

45. Collins PJ, Horowitz M, Maddox A, et al. (1996) Effects of increasing solid component size of a mixed solid/liquid meal on solid and liquid gastric emptying. Am J Physiol 271, G549-G554.

46. Karamanlis A, Chaikomin R, Doran S, et al. (2007) Effects of protein on glycemic and incretin responses and gastric emptying after oral glucose in healthy subjects. Am J Clin Nutr $\mathbf{8 6}$, 1364-1368. 
47. Muniyappa R \& Sowers JR (2013) Role of insulin resistance in endothelial dysfunction. Rev Endocr Metab Disord 14, $5-12$.

48. Cai H \& Harrison DG (2000) Endothelial dysfunction in cardiovascular diseases: the role of oxidant stress. Circ Res $\mathbf{8 7}$, 840-844.

49. Brouwers O, Niessen PM, Haenen G, et al. (2010) Hyperglycaemia-induced impairment of endotheliumdependent vasorelaxation in rat mesenteric arteries is mediated by intracellular methylglyoxal levels in a pathway dependent on oxidative stress. Diabetologia 53, 989-1000.

50. Adolphe JL, Drew MD, Huang Q, et al. (2012) Postprandial impairment of flow-mediated dilation and elevated methylglyoxal after simple but not complex carbohydrate consumption in dogs. Nutr Res 32, 278-284.

51. Davalos A, Miguel M, Bartolome B, et al. (2004) Antioxidant activity of peptides derived from egg white proteins by enzymatic hydrolysis. J Food Prot 67, 1939-1944.

52. Nimalaratne C, Lopes-Lutz D, Schieber A, et al. (2011) Free aromatic amino acids in egg yolk show antioxidant properties. Food Chem 129, 155-161.

53. Needleman P, Turk J, Jakschik BA, et al. (1986) Arachidonic acid metabolism. Annu Rev Biochem 55, 69-102.

54. Menke A, Casagrande S, Geiss L, et al. (2015) Prevalence of and trends in diabetes among adults in the united states, 19882012. JAMA 314, 1021-1029. 